\title{
Frontières
}

\section{La pratique du barebacking et le VIH/sida}

\section{Godelieve Brusselaers et Jocelyne Saint-Arnaud}

Volume 18, numéro 2, printemps 2006

La mort dans tous ses états

URI : https://id.erudit.org/iderudit/1073223ar

DOI : https://doi.org/10.7202/1073223ar

Aller au sommaire du numéro

Éditeur(s)

Université du Québec à Montréal

ISSN

1180-3479 (imprimé)

1916-0976 (numérique)

Découvrir la revue

Citer cet article

Brusselaers, G. \& Saint-Arnaud, J. (2006). La pratique du barebacking et le VIH/sida. Frontières, 18(2), 57-62. https://doi.org/10.7202/1073223ar

\section{Résumé de l'article}

La pratique du barebacking est apparue dans les milieux gais américains vers 1995. Au départ, elle était le fait d'homosexuels séropositifs et séronégatifs qui, par le biais d'Internet, organisaient des rencontres dont le but était la recherche de rapports sexuels sans protection. Un témoignage illustre tout le drame humain sous-jacent à la recherche de partenaires sexuels qui acceptent volontairement de risquer leur santé, sinon leur vie. Ce phénomène tirerait son origine d'un mouvement de protestation contre les effets discriminatoires qu'ont eus les interventions préventives de la santé publique sur le groupe cible. Différentes solutions sont présentées. Les solutions coercitives sont rejetées au profit d'une approche humaniste individualisée. 


\section{Résumé}

La pratique du barebacking est apparue dans les milieux gais américains vers 1995. Au départ, elle était le fait d'homosexuels séropositifs et séronégatifs qui, par le biais d'Internet, organisaient des rencontres dont le but était la recherche de rapports sexuels sans protection. Un témoignage illustre tout le drame humain sous-jacent à la recherche de partenaires sexuels qui acceptent volontairement de risquer leur santé, sinon leur vie. Ce phénomène tirerait son origine d'un mouvement de protestation contre les effets discriminatoires qu'ont eus les interventions préventives de la santé publique sur le groupe cible. Différentes solutions sont présentées. Les solutions coercitives sont rejetées au profit d'une approche humaniste individualisée.

Mots clés: barebacking - VIHISida homosexuels.

\section{Abstract}

Barebacking first appeared in American gay circles around 1995. Initially, barebacking was used by seropositive and seronegative homosexual men who planned dates on the net with the intention of having sexual intercourses without any protection. The story, related in this article, shows the human drama underlying the search for sexual partners who accept to place their health, and even their life, at risk. That phenomenon would come from a protest movement against the discriminatory effects of public health preventive interventions on the target population. Some solutions are brought about. Coercive ones are rejected in favour of a humanist individualized approach.

Keywords: barebacking - HIVIAids homosexuals.

\section{N T E R V E N T I O N}

\section{LA PRATIQUE DU BAREBACKING ET LE VIH/SIDA}

Godelieve Brusselaers, infirmière.

Jocelyne Saint-Arnaud, Ph. D. professeure, Faculté des sciences infirmières, Université de Montréal.

Bien que les différents dispositifs d'information et de prévention sur le sida aujourd'hui disponibles soient multiples et variés, le discours collectif tend à les décrire comme très réduits, voire inexistants. C'est comme si, pour de très nombreux hommes gais, le dispositif de prévention du sida dans son ensemble était devenu invisible. L'une des croyances qui prévaut actuellement dans le milieu gai indique que beaucoup d'hommes adoptent des pratiques qui comportent un risque majeur. Deux termes peuvent résumer cette croyance: relapse et bareback. Le terme relapse indique l'idée d'un relâchement des comportements de prévention et le bareback est synonyme d'abandon volontaire des mesures de prévention. Selon Le Talec (2001), pour expliquer ces prises de risques, nous pouvons évoquer le changement de contexte engendré par l'évolution des traitements. Limage du sida, en tant que maladie, s'est modifiée.

Pratiquer le barebacking, c'est choisir délibérément d'avoir des relations sans condom. Apparu dans le milieu gai aux États-Unis vers 1995, aux États-Unis, le barebacking peut être considéré selon Scarce (1999) comme le refus de toute mesure de prévention dans les relations sexuelles. Ce refus serait revendiqué et médiatisé par les barebackers, qui se retrouvent au sein d'une sous-culture gaie. Il s'est développé sous la forme de réseaux, essentiellement par le biais d'Internet: sites spécialisés, petites annonces, agendas de soirées. Il existe un langage et des codes propres au barebacking.

Le Talec (2000) affirme que si le bareback a été initialement décrit dans un contexte homosexuel, il concerne aujourd'hui tout le monde, hommes et femmes, toutes sexualités confondues. La question de l'abandon des mesures de prévention du sida et des MTS se pose à chacun d'entre nous. Entre autres, Welzer-Lang (1999) souligne que la présence du barebacking concerne des personnes qui en font le choix délibéré, mais peut aussi attirer et séduire des hommes et des femmes mal informés ou prêtes à renoncer au préservatif pour qu'une rencontre se concrétise. Mais cet auteur affirme aussi qu'on ne peut limiter les débats sur le bareback aux débats sur le choix sexuel des adultes consentants, agissant librement. Selon Mendes-Leite (1995), il faut aussi mettre en parallèle les constats d'isolement social, de misère affective, d'homophobie vécue et intériorisée et le risque de contamination auquel sont exposés des jeunes cherchant une sexualité à tout prix.

Pour illustrer la problématique, des hommes gais décrivent des pratiques extrêmes qui sont de plus en plus courantes dans les saunas. Ils rapportent qu'à maintes reprises des hommes veulent retirer leur condom pendant la relation. Ils sont témoins de 
la pratique de relations complètes entre hommes sans aucune protection. Des rencontres s'organisent sur Internet avec comme but affiché des relations sexuelles entre séropositifs sans utilisation de condoms. La conversation suivante a été recueillie par l'une des auteures. Un ami homosexuel s'est confié à elle à la suite d'une rencontre qu'il a eue avec un homme séropositif avec qui il avait échangé des propos lors d'une séance de clavardage:

qui vont se donner encore une raison pour descendre dans la déchéance... Je l'ai bien vu, moi, avec ce garçon sidéen que j'ai rencontré... si beau et si gentil et pourtant avec le désir de chercher un venin qui le rendrait superpuissant... Les ténèbres, elles sont là; les ténèbres et aussi la déchéance humaine et en abondance... Moi, je l'ai rencontré ce garçon sidéen et si tu veux savoir plus, je l'ai embrassé sur les lèvres. Et lui était étonné... mais j'ai senti que mon amour pour les êtres est plus fort que tout et lui, il a eu un mouvement de saisie... Heureusement pour moi. Et il s'est mis à être gentil et là... je me suis rendu compte que si je l'avais vu au sauna, sans savoir qu'il était séropositif, sûrement j'aurai voulu tout faire avec lui... Mais hélas, on ne peut plus rien pour lui et je lui ai pas plu sexuellement... Il cherchait une bête et il s'est juste accommodé de ma poitrine et accepté ma tendresse... Pourtant il m'a dit que des mecs qui cherchaient le sexe voulait le faire avec lui sans condom en sachant qu'il était HIV positif... Il était dans cette dualité vie-mort et j'ai vu en lui un miroir comme une partie de moi reflétée... mais lui c'était pire après tout ce cauchemar qu'il m'avait raconté... Il cherchait et cherche encore l'Amour d'une mort bien méritée... il cherche un dard pour se crever finalement et arrêter tout, mourir... J'ai vu le suicide en lui que moi j'ai déjà ressenti... et pourtant la vie en moi était plus forte... sinon je crois que j'aurais attrapé ce virus et que je l'aurai attiré avec mon magnétisme personnel... J'ai voulu arrêter d'emblée... plus de sauna... plus de sexe anonyme... plus d'expositions inutiles et foutaises... Tout finit là parce qu'il avait l'air si sain et pourtant quand je l'ai serré dans mes bras... j'ai serré un peu la mort... Je ne serai pas un individu... je serai un mort-vivant... un zombie... un être qui a eu si mal et qui est en train d'agoniser jour après jour... Dommage que je ne le reverrai pas puisqu'il n'a pas eu ce qu'il recherchait avec moi... Lui, il cherche

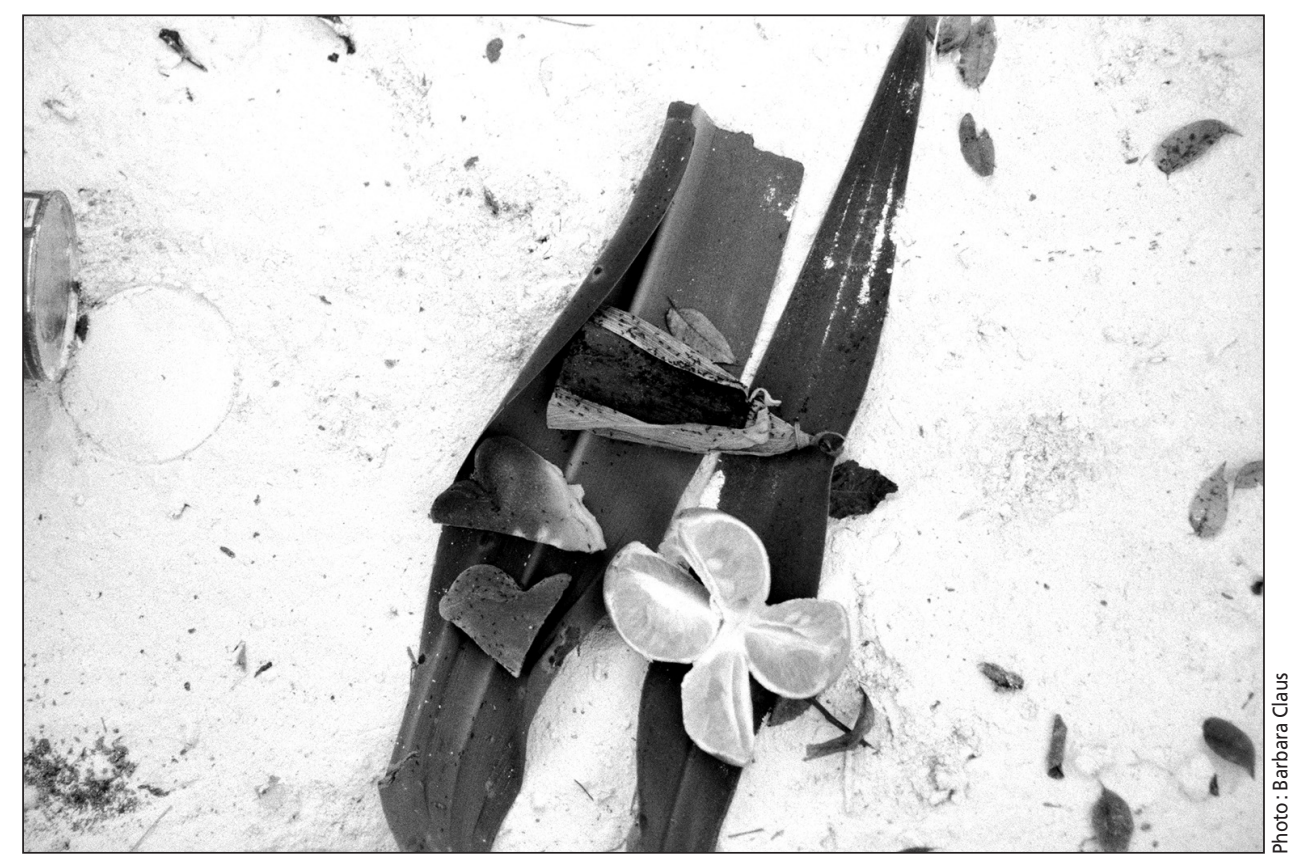

un genre d'anesthésie pour lui faire oublier... Unir vie et mort à travers une orgie n'est pas la magie... c'est la voie de notre propre ombre intérieure qui nous noue les horizons en nous proposant de mourir... et de nous libérer comme ça... alors qu'elle sait très bien qu'elle veut nous dévorer.

Ce témoignage suscite différents questionnements. Sans entrer dans une démarche psychanalytique approfondie, il est facile de reconnaître ici un certain nombre de tensions: attirance/rejet, amour/haine, vie/mort, qui s'entremêlent dans un drame humain hautement personnel, mais qui nous interpelle en tant qu'humain et que citoyen. Quelle attitude adopter face à cette pratique? N'est-il pas du devoir des organismes de santé publique d'intervenir? Une intervention coercitive est-elle appropriée? Les approches préventives traditionnelles seraient-elles suffisantes et surtout efficaces?

\section{POUR COMPRENDRE LA PROBLÉMATIQUE}

Carson (1998) affirme que le terme de bareback n'est pas neutre; il évoque implicitement la séropositivité et le risque de (sur)contamination. Cet auteur nous explique que ces groupes de barebackers ne sont pas constitués d'hommes naïfs qui ont oublié les ravages du VIH dans leur propre communauté, mais plutôt d'hommes séropositifs ou assumant le risque de le devenir.

Selon Garnier et Lambeaux (2000), le safe-sexe (sexe sans risques, rapports protégés) est prôné par les associations travaillant sur les comportements à risque et leur prévention. Mais le barebacking s'exerce entre individus sérodiscordants, c'est-à-dire séropositifs et séronégatifs, ou entre séropositifs contaminés par des souches différentes du virus. Pour ces derniers, leur comportement est fondé sur le fait qu'ils estiment que le VIH n'est pas leur identité, qu'il n'y a plus de risques à cause de l'utilisation des nouveaux traitements et que la surcontamination n'existe pas. Les personnes séropositives estiment que les discours de la santé publique sur le sida ont brimé leur sexualité en imposant des normes qui visaient un nouveau contrôle social et sociétal. Ils estiment pouvoir faire ce qu'ils veulent de leur corps. Ils rappellent que le barebacking est pratiqué entre personnes consentantes et pleinement informées des risques qu'elles encourent. Enfin, ils refusent toute ingérence de l'État dans leurs comportements sexuels.

En ce qui concerne les personnes séronégatives qui se prêtent à cette roulette russe,
LES PERSONNES SÉROPOSITIVES

ESTIMENT QUE LES DISCOURS DE LA SANTÉ PUBLIQUE SUR LE SIDA ONT BRIMÉ LEUR SEXUALITÉ EN IMPOSANT DES NORMES QUI VISAIENT UN NOUVEAU CONTRÔLE SOCIAL ET SOCIÉTAL. 


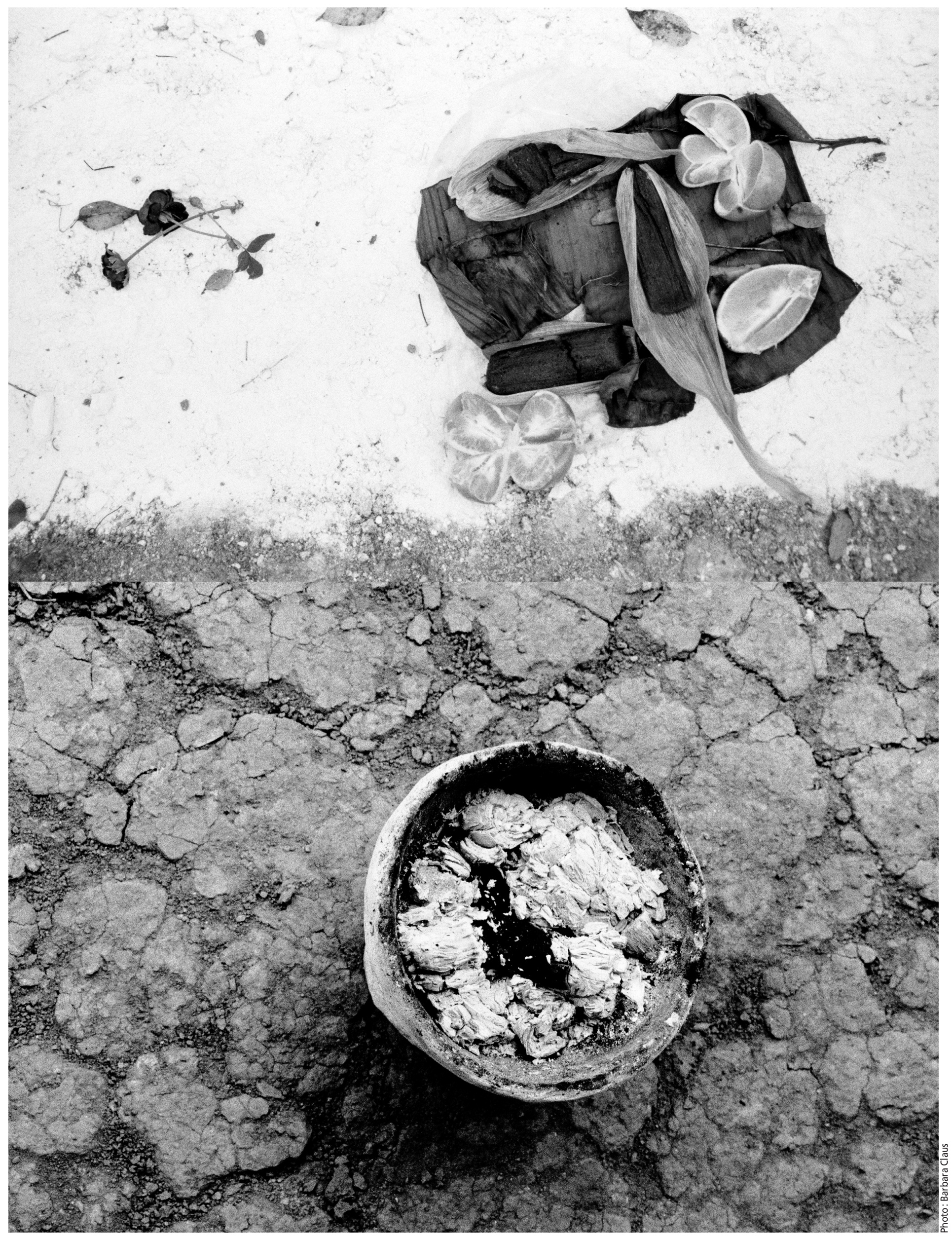


Garnier et Lambeaux (2000) affirment que les choses sont différentes. Être gai, c'est le plus souvent être rejeté par les parents et n'être rien pour la société. Pour beaucoup de jeunes, le fait d'être séropositifs les aide, selon ces auteurs, à faire leur coming out dans la famille et à y recevoir de l'attention. Ils ne sont «plus rien » pour la société, mais ils sont séropositifs. C'est ce qui crée leur identité.

Selon Le Talec (2000), il semble y avoir une relation de cause à effet entre l'émergence du barebacking et l'arrivée des antiprotéases et des trithérapies. Aujourd'hui, l'amélioration des résultats et la possibilité de recourir à des traitements post-exposition créent un contexte qui peut expliquer que certains gais choisissent d'abandonner le préservatif. Scarce (1999), pour sa part, affirme que l'érotisation du risque et du virus a pu être constatée entre partenaires barebackers. Selon lui, l'intimité recherchée lors de sodomies non protégées confère une dimension allégorique à la contamination et à l'invasion virale. Le partenaire qui recherche la contamination est dit bug chaser et l'infection est dénommée "fécondation». Le partenaire contaminant est dit gift giver et il assume la "paternité » de la contamination. Scarce (1999) conclut que pour ces hommes, la séroconversion est devenue un rituel d'adoption formulé avec des métaphores de la grossesse, plutôt que le fruit du hasard.

\section{PUNIR LES COUPABLES?}

Ceux qui proposent de criminaliser les actes de personnes séropositives qui risquent d'infecter d'autres personnes soutiennent que cela est nécessaire pour punir, dénoncer une conduite répréhensible, réhabiliter les contrevenants, prévenir le tort à autrui et dissuader les contrevenants et le public de se conduire de la sorte. Lesquels de ces objectifs sont importants? Les lois et les interventions des organismes de santé publique peuvent-elles atteindre ces buts sans brimer l'autonomie individuelle et limiter l'expansion de la maladie?

La santé publique ne vise pas à punir, contrairement au droit criminel. Mais est-ce l'objectif principal: punir ceux qui en infectent d'autres? Puisque la punition n'est justifiée que si elle est méritée, les poursuites ne seraient justifiées que si la conduite est clairement blâmable au plan moral. Les sanctions criminelles sont habituellement réservées aux gens qui font du tort à d'autres de façon intentionnelle ou insouciante. Cependant, la punition est fréquemment question de vengeance et favorise les préjugés sociaux et la désinformation. Les personnes vivant avec le VIH sont souvent jugées responsables de leur état, surtout si ce sont des homosexuels, des prostituées ou des utilisateurs de drogue.
Les gens sont alors punis pour ce qu'ils sont autant que pour ce qu'ils ont fait. Or, la discrimination et la stigmatisation à l'égard des communautés touchées par le VIH nuisent déjà aux efforts de prévention du VIH. Une approche qui se prévaut du pouvoir de l'État de punir, plutôt que d'aborder les raisons pour lesquelles certains s'adonnent à des activités à risque, aggrave le problème. La moralisation et la recherche de boucs émissaires ont déjà empiré l'épidémie de VIH.

\section{RESPONSABILITÉS PARTAGÉES}

En nous basant sur l'article de Hans-Balz Peter (2003) intitulé «La dimension éthique», nous pouvons dire que nous sommes en présence d'une relation socioéthique que nous décrirons comme suit: professeurs, etc.) la responsabilité de signaler les cas d'infection qu'ils soupçonnent;

3. accorder aux autorités sanitaires des pouvoirs relativement vastes pour protéger la santé publique.

Dans le registre le plus coercitif, il s'agit de lois quasi criminelles. Les responsables de la santé publique peuvent imposer un examen et un traitement à une personne soupçonnée d'avoir une maladie, et ordonner une conduite pour éviter l'infection d'autres gens. Par exemple, un responsable de la santé publique peut interdire à quelqu'un d'avoir des rapports sexuels sans protection ou l'obliger à révéler sa séropositivité à ses partenaires. Dans toutes les provinces, les autorités de la santé ont le pouvoir de faire détenir des gens (généralement à l'hôpital) pour prévenir

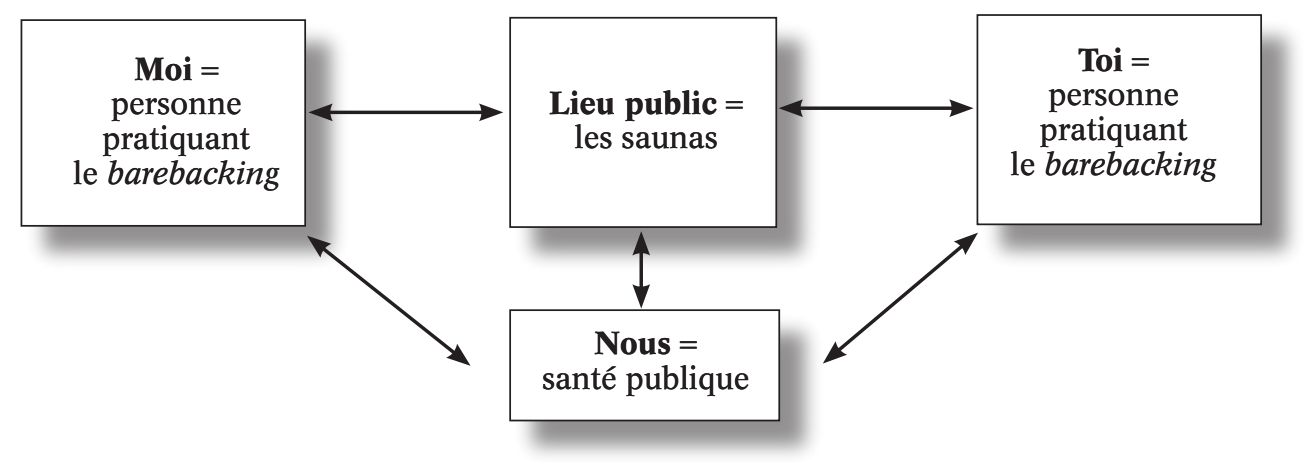

Une telle responsabilité collective inclut et dépasse la responsabilité des individus impliqués et interpelle les organismes de santé publique. Dans cette perspective, différentes instances doivent prendre leurs responsabilités pour éviter des conséquences néfastes, voire dramatiques pour la santé du genre humain. Il s'agit plus précisément d'appliquer une éthique utilitariste, sans juger l'individu pour ses actes. Au lieu de cibler les caractéristiques de l'agent qui le stigmatisent et lui portent préjudice, on voudra centrer l'intervention sur les conséquences à court et à long terme de ces pratiques: la transmission du VIH et l'expansion de l'épidémie. Une telle approche éthique oblige l'individu qui pratique le barebacking à réfléchir aux conséquences que ses actions auront sur les autres.

\section{LA MISSION DES ORGANISMES DE SANTÉ PUBLIQUE}

Les lois sur la santé publique varient d'une province à l'autre, mais elles ont partout les mêmes fonctions:

1. classifier les maladies transmissibles et préciser quelles règles s'appliquent à chacune;

2. imposer aux personnes atteintes l'obligation de recevoir un traitement médical, et à d'autres (médecins, la propagation de maladies (Droit criminel et VIH/sida, mars 1999).

Selon Mann (1998), dans le contexte du sida, la santé publique a appris que la discrimination envers les personnes séropositives ou atteintes du sida est contre-productive. Il affirme qu'il existe une relation entre santé publique et droits de la personne. Les atteintes aux droits de la personne ont des conséquences sur la santé, c'est-à-dire des effets négatifs sur le bien-être physique, mental et social. Au fur et à mesure de sa maturation et de son évolution, l'épidémie du VIH s'est focalisée, au sein de chaque communauté, sur les groupes qui étaient déjà l'objet de discrimination et qui étaient marginalisés et stigmatisés par la société. La vulnérabilité à l'épidémie a été associée à une extension de la reconnaissance des droits de la personne, plus précisément à la reconnaissance des droits des gais. Pour cette raison, selon Mann (1998), les efforts de prévention contre le sida doivent maintenant selon Mann (1998) dépasser les moyens traditionnels d'éducation et de services pour s'intéresser aux questions relatives aux droits de la personne. Autrement dit, la simple prévention n'est pas suffisante. Il faut agir contre la discrimination pour intervenir de manière efficace contre la transmission du virus. 


\section{PISTES DE SOLUTIONS}

Dans les milieux de la santé publique, l'inquiétude est très répandue face à un recours aux sanctions pénales contre des gens qui ont des activités comportant un risque de transmission du VIH et face à des propositions de modifier le Code criminel pour créer une infraction spécifique au VIH. En particulier, on craint qu'une infraction criminelle spécifique contribue à stigmatiser davantage les personnes vivant avec le $\mathrm{VIH} /$ sida et d'autres maladies transmissibles sexuellement. Non seulement est-il improbable que des poursuites soient efficaces dans la majorité des cas de transmission du VIH, mais une mesure sociale aussi coercitive pourrait faire plus de tort que de bien. L'histoire montre que les politiques punitives et coercitives nuisent aux objectifs de la santé publique. Il est peu probable que les poursuites empêchent les activités à risque et une stratégie de réduction des méfaits apparaît plus appropriée.

Le modèle de la prévention, élaboré à partir d'une problématique qui tient compte de facteurs de risque définis collectivement, soulève une double question: comment et dans quelle limite rassembler les individus autour de cette perception collective d'un danger? (Dodet et al., 1998.) Les responsables de la santé publique doivent trouver un équilibre entre les deux objectifs prioritaires de la santé publique moderne: promouvoir et protéger la santé publique, d'une part, et promouvoir et protéger les droits de la personne et la dignité humaine, d'autre part. Nous aurons peut-être plus de chances de modifier les comportements en cherchant les raisons pour lesquelles certains s'adonnent au barebacking. Alors que le recours aux sanctions pénales contribue à faire en sorte que des personnes vivant avec le VIH et pratiquant le barebacking soient considérées comme des criminels et des dangers publics, les interventions personnalisées et respectueuses de la confidentialité comportent moins de risques de stigmatisation. Le barebacking apparaît en effet comme une protestation contre la stigmatisation et le moralisme ou encore comme un acte de désespoir et d'hostilité face à la vie, une forme de suicide qui peut sembler très rationnelle aux yeux de ceux qui le pratiquent. Il faut donc adapter les interventions en fonction des pratiques extrêmes.

Le but qui devient alors prioritaire est d'informer, former et conseiller les populations sur leur condition de santé et sur des moyens de prévention et de protection pertinents (Saint-Arnaud, 2002). Il reste à trouver, au sein des institutions, un espace de dialogue, où la morale sexuelle peut aussi avoir sa place, sans pourtant empêcher toute discussion. Cet objectif constitue très certainement l'un des nouveaux défis majeurs de la santé publique: arriver par des méthodes préventives et des campagnes de promotion à changer les attitudes et les comportements pour les rendre plus responsables. Dans cette optique, il paraît urgent, selon Le Talec (2000)

- de faire prendre conscience de l'importance de la prévention, de donner envie de se protéger,

- de réaliser des campagnes de prévention dans les médias grand public,

- d'améliorer les outils de prévention et l'accès à ces moyens (exemple: préservatifs gratuits),

- de se débarrasser des contraintes moralisantes en matière de prévention,

- de développer l'écoute,

- de communiquer au sujet de l'homosexualité, des modèles de vie gaie, et d'étudier les comportements et les pratiques à risque,

- de développer la créativité,

- de travailler sur les liens entre prévention et relations affectives.

Ce qu'il ne faut pas faire, toujours selon

Le Talec (2000):

- continuer, ne rien changer,

- baisser les bras, arrêter les campagnes de prévention,

- banaliser le sida, faire croire que tout va bien,

- adopter un discours moralisateur et contrôleur.

Une approche novatrice pourrait, par exemple, amener la création de lieux interinstitutionnels ouverts, consacrés à la prévention et à la santé en général des gais. Il importe aussi de pouvoir exercer un certain contrôle dans ces établissements sur le plan de l'hygiène et de la prévention, mais sans que ce pouvoir soit coercitif, discriminatoire et stigmatisant.

\section{PRINCIPES D'UNE RELATION THÉRAPEUTIQUE}

Fruit de la collaboration de l'Association canadienne des soins de santé, de l'Association médicale canadienne, de l'Association des infirmières et infirmiers du Canada et de l'Association catholique canadienne de la santé, la Déclaration conjointe sur la prévention et le règlement de conflits éthiques entre les prestateurs de soins de santé et les personnes recevant les soins (1999) présente les principes d'une relation thérapeutique. En voici quelques extraits:

Les besoins, les valeurs et les préférences de la personne qui reçoit les soins devraient être la considération primordiale dans la prestation de soins de santé de qualité. Idéalement, les décisions en matière de soins de santé seront le fruit d'un consensus entre la personne qui reçoit les soins et tous ceux qui prennent part à ces soins. [...] Les bonnes relations thérapeutiques s'appuient avant tout sur les besoins et les choix éclairés de la personne qui reçoit les soins. De telles relations se fondent sur le respect et sur le caractère mutuel du don et de l'acceptation. [...] Une communication ouverte s'impose dans un contexte de confidentialité et de protection de la vie privée. Il faut encourager tous ceux qui participent à la prise de décisions à exprimer leur point de vue. [Nous devons nous assurer] de comprendre les besoins, les valeurs et les préférences de la personne qui reçoit les soins.

<http://cna-aiic.ca/cna/>, 1999.

Il est primordial que la personne qui reçoit les soins comprenne l'information transmise, il faut lui fournir le temps, le soutien et les occasions nécessaires pour participer pleinement aux décisions qui la concernent.

À notre avis, une intervention préventive, respectueuse de la personne, effectuée individuellement et délicatement, s'avérerait plus efficace que des interventions étatiques qui, en s'adressant au groupe, le stigmatisent ainsi que les individus qui en font partie.

Selon Defert (1990):

le safer sex, la sexualité sans risque, c'est une sexualité; à l'extérieur, on entend trop cela comme une asexualité. Se dire séropositif, c'est souvent pour l'extérieur se faire connaître comme vivant une asexualité. Si on fait de la sexualité protégée une idéologie officielle et non à nouveau l'objet d'un débat sur la sexualité, on risque d'imposer un nouveau silence sur la vérité des pratiques sexuelles aujourd'hui. On risque de mauvaises surprises à court terme si on transforme le safer sex en langue de bois militante. Je crois que les homosexuels ne peuvent pas réduire tous leurs enjeux à la bonne image de contrôleur de l'épidémie, car si cette image redevenait un leurre, cela coûterait cher à la fois en vies et en réactions sociales. (1990, p. 60)

Le danger que Defert (1990) soulignait semble toujours d'actualité; les contrôleurs de l'épidémie sont aujourd'hui à bout de force et désabusés. Pour sortir de cette impasse tout en continuant de promouvoir la santé publique, la langue de bois militante n'est plus de mise, d'autant plus que certains hommes gais, adeptes du barebacking, libèrent leur sexualité des contraintes nées du sida en assumant les risques qui découlent d'un tel choix. 


\section{Bibliographie}

DEFERT, D. (1990). "L'enjeu des gais L'homosexualisation du sida", Gai Pied Hebdo, n ${ }^{\circ}$ 446, 29 novembre, p. 60-63.

DODET, B., C. PERROTIN et L. VALETTE (1999). «Santé publique et éthique universelle ou comment concilier les tensions entre le bien de la personne et le bien commun?", Les pensières, Veyvrier-du-Lac, juin 1998, Paris, Elsevier.

MANN, J. (1998). "Santé publique: éthique et droits de la personne», Santé publique, vol. $10, \mathrm{n}^{\circ} 3$, p. 239-250.

MENDES-LEITE, R. (1995). «Combien ou comment? Le multipartenariat sexuel et la gestion des risques de transmission du sida ", Quel corps, $\mathrm{n}^{\text {os }} 47-48-49$, p. 70-91.
PETER, H.-B. (2003). «La dimension éthique: Le suicide est-il justifiable à certaines conditions?», chapitre 5 (p. 69-96) dans E. Aebischer, A. Bondolfi, A. Bopp, D.A. Castelli, M. Grünewald, F. Gutzwiler, P.-A. Michaud, K. Michel, P. Mösli, E. Murer, C. Odier, M. Perret-Catipovic, H.-B. Peter, H. Poltier, J. Rutgers-Cardis et J. et H. Saver. (2003). Suicide. La fin d'un tabou?, Genève, Labor et Fides.

SCARCE, M. (1999). La fin du safer sex, Traduction de C. Martet, Têtu, $\mathrm{n}^{\circ}$ 33, avril 1999.

ST-ARNAUD, J. (2002). «La fin justifie-t-elle les moyens? », L'infirmière canadienne, avril, vol. 3 et 4, p. 4, 6-8.

WELZER-LANG, D. (1999). Bisexualités masculines et prévention sida, projet de recherche présenté à l'Agence nationale de recherches sur le sida, France.

\section{Sites Web}

CARSON, G. (1998). Psychotherapy with Men who Bareback, communication au Congrès de la California Psychological Association, Pasadena, Californie. <www. gaytherapy.com>.

Déclaration conjointe sur la prévention et le règlement de conflits éthiques entre prestataires de soins de santé et personnes recevant les soins. <http://cna-aiic.ca/cna/>.

Droit criminel et VIH/sida, mars 1999. $<$ http://www.aidslaw.ca $>$.

GARNIER, E. et S. LAMBEAUX (2000). «Du bûcher au barebacking, quelle réalité pour les homo?». <http://www.ainfos.ca $>$.

LE TALEC, J.Y. (2000). «Le risque du sexe, entre rumeur et réalité. La prévention de la transmission du VIH chez les homosexuels masculins, état des lieux du dispositif à Paris, établi entre janvier et juillet 2000 », Rapport de recherche, septembre $2000 .<\mathrm{http}: / / \mathrm{www}$. multisexualites-et-sida.org $>$.

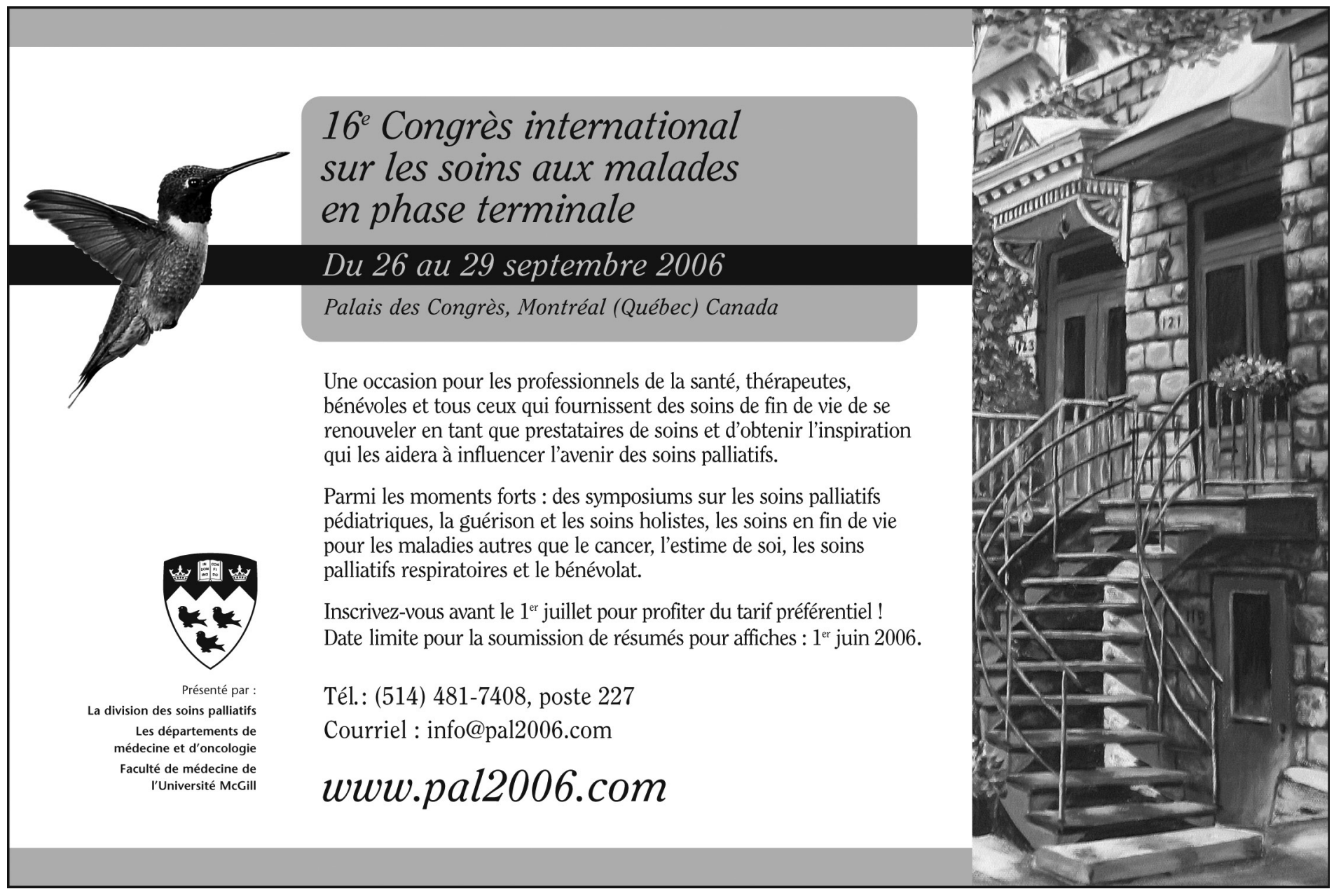

